\title{
La oportunidad de convertirme en una investigadora del quehacer educativo: vivencias de una investigación-acción
}

\author{
Luz María Cárcamo López"11 \\ División de Educación Básica \\ Centro de Investigación y Docencia en Educación \\ Universidad Nacional \\ Heredia, Costa Rica \\ Imcarcamogo@yahoo.es
}

\section{Resumen}

Convertirse en un investigador desde una propuesta sustentada en la investigación-acción es transitar por el cuestionamiento, la transformación, la toma decisiones reflexivas aceptando la responsabilidad de las mismas, es estar abierto al aprendizaje, la búsqueda constante, la incertidumbre, la curiosidad, la creatividad y la crítica, pero sobre todo amar lo que se hace en aras de en conjunto generar propuestas de acción oportunas, coherentes con la realidad, fundamentadas en la mejor información disponible y sujetas a la transformación permanente derivada de la reflexión. El presente artículo representa la síntesis de un experiencia de investigaciónacción sustentado en la premisa ontológica de que además de ser inevitable el cambio reflexivo-activo es necesario, en tanto constituye el medio para enriquecer el saber y el hacer pedagógico, basada en una constante construcción, reconstrucción y transformación de la realidad mediante el cuestionamiento crítico y la reflexión

\footnotetext{
${ }^{11}$ Licenciada en Educación Pre-Básica graduada en la Universidad Pedagógica Nacional Francisco Morazán (Honduras). Tiene experiencia docente en educación básica y pre-básica en el sector público y privado hondureño. Actualmente labora como docente en el Centro de Educación Básica Luis Landa en Honduras. Máster en Pedagogía con énfasis en Desarrollo y Atención Integral de la Primera Infancia, de la Universidad Nacional de Costa Rica.
} 
permanente. En especial cuando el diálogo, la observación, la reflexión y el cuestionamiento son las herramientas utilizadas, que permiten poco a poco desarrollar conciencia acerca de nuestros propios actos en el aula.

Palabras claves. Investigación, transformación, reflexión y cuestionamiento.

Keywords. Investigation, transformation, reflection and questioning.

\section{Introducción}

Cuando ya no somos capaces de cambiar una situación, nos encontramos ante el desafío de cambiarnos a nosotros mismos.

Victor Frankl (2011)

Convertirse en un investigador desde una propuesta sustentada en la investigación-acción es transitar por el cuestionamiento, la transformación, la toma decisiones reflexivas aceptando la responsabilidad de las mismas, es estar abierto al aprendizaje, la búsqueda constante, la incertidumbre, la curiosidad, la creatividad y la crítica, pero sobre todo amar lo que se hace en aras de en conjunto generar propuestas de acción oportunas, coherentes con la realidad, fundamentadas en la mejor información disponible y sujetas a la transformación permanente derivada de la reflexión.

El presente artículo representa una nueva oportunidad para compartir la vivencia de un proceso de investigación, acción y reflexión, que se desarrolló en un Jardín de Niños Público, con un grupo de 19 niños y 14 niñas del ciclo de Transición y la docente a cargo de este grupo titulada Intervención docente: estrategias pedagógicas que responden a los niños, las niñas sus intereses y necesidades. 
Para ello, nos permitimos proponer y vivir este proceso asumiéndonos como seres capaces de saber, que sabemos y no sabemos, que podemos saber mejor lo que ya sabemos y de saber de lo que aún no sabemos (Freire, 2007), partimos de la pregunta ¿Qué aspectos pedagógicos deben considerarse para que la intervención docente responda a los intereses y necesidades de los niños (as) de manera que se potencie su desarrollo integral? buscamos respuestas por medio de ocho encuentros cuyo componente principal fue la acción y la reflexión, reconociéndonos como seres flexible, consciente de las propias responsabilidades y conocimientos, asumiendo nuevos roles, permitiéndonos acompañar y ser acompañados por otros (as), experimentar armonía entre lo que se vive, hace, piensa y necesita. Sin lugar a duda fueron los espacios para la observación, el cuestionamiento, el análisis, la acción y la reflexión sobre nuestras actuaciones, roles asumidos, permitidos y las percepciones del acto educativo los hallazgos más importantes en la búsqueda de respuestas a la pregunta planteada.

A la luz de la dinámica generada a lo largo de todo este proceso se puede decir entonces que, para crear una dinámica de aula respetuosa de los niños (as) sus intereses y necesidades de desarrollo, primeramente se tiene que tener claridad entre ¿qué y cuáles son?, esa fue la primera puerta de entrada conocer a los niños (as), lo que sienten, piensan, hacen, desean y pueden hacer, por medio de la proposición de talleres porque parecía ser el método más cercano al movimiento natural de ellos (as) y sus deseos de aprender haciendo, con la intensión de entender sí mediante esto, se podría generar una comprensión y percepción diferente de los rol que hemos asumido; construyendo y validando juntos (docentesestudiantes) nuevas estrategias pedagógicas donde todas (as) se sintieran tomados en cuenta y puedan participar de manera activa. Posteriormente se presentó la posibilidad de un giro, donde la intensión fue ver ¿cómo la docente organiza su clase y bajo qué criterios se planificaban las actividades? y ¿cuál es su rol?, creando por medio de esto espacios para diálogo, la reflexión, la modificación de roles y rutinas. Sin embargo surgió la necesidad de replantear 
algunos elementos y construir conjuntamente entre los docentes criterios para ser tomados en cuenta antes de organizar las actividades que se proponen, con la intensión de generar una dinámica de aula diferente. Llegando al punto de organizar juntas la práctica pedagógica, sustentada en las construcciones y aprendizajes logrados a lo largo de todo el proceso.

\section{El camino transitado siendo investigadora al investigar: las construcciones}

No hay enseñanza sin investigación, ni investigación sin enseñanza

Paulo Freire (1997)

De la certeza hacia la incertidumbre constituyó el punto de partida de este proceso de investigación- acción, desarrollado en el marco del paradigma naturalista, pues poco se aprende cuando se parte sólo de respuestas concebidas como verdades absolutas e incuestionables. Es por ello, que se encaminaron los pasos a la vivencia de este proceso investigativo, cuya intencionalidad se centró en la intervención docente: estrategias pedagógicas que responden a los niños (as) sus intereses y necesidades, en tanto fue la reflexión de la docente a cargo del grupo y la mía como docente investigadora la que permitió ir dando forma a los conocimientos, transformaciones y acciones. Asumiendo la investigación-acción, como una intervención en la práctica con la intención de ocasionar una mejora, buscamos una educación verdadera desde la praxis, reflexión, acción y transformación (Latorre, 2005).

Se inició una aventura teñida de sentimientos, miedos, angustias y emociones, sin pensar en la transformación que este proceso generaría, como se mencionó anteriormente guiada por la pregunta ¿Qué aspectos pedagógicos deben considerarse para que la intervención docente responda a los intereses y necesidades de los niños (as) de manera que se potencie su desarrollo integral? Cabe mencionar que enfrentar un cambio de paradigma resultó ser un 
ejercicio difícil, por las rupturas epistemológicas, heurísticas, ontológicas y axiológicas que implica el contraste con nuestro marco de referencia más coherente con el positivismo. Genera angustia, enfrentarse a una transformación profunda, darse la oportunidad de ser falible y equivocarse, ser héroe y ser humano al mismo tiempo, comprender el "enseñar y aprender" de modo diferente, crear un tiempo y espacio para la producción de conocimientos. Progresivamente, va surgiendo la necesidad de una actitud cada vez más abierta, de una revisión crítica del hacer, con el propósito de crear una educación más liberadora, no solo para las y los estudiantes, sino también para las educadoras, es decir, remirar la práctica a la luz para abrirse a nuevas posibilidades (Peralta, 2002).

Como investigadora en este proceso, me permití proponer a la docente del grupo y a los niños (as) construir espacios lúdicos que facilitaran el transitar por los caminos de la acción, la reflexión, la conversación, el aprendizaje, la sistematización y la transformación. Como personas adultas a cargo, el análisis de estas vivencias con el grupo permitió progresivamente asumir una conciencia crítica en torno al quehacer educativo, identificando aciertos, errores y dudas. Lo que nos ubicó en un universo pleno de alternativas, donde educar se constituye en un proceso que transforma espontáneamente. Nuestras creencias, valores, conocimientos previos incidieron en nuestro hacer, sentir y pensar educativo y nos llevó a la posibilidad de desaprender y aprender a aprender juntos experimentando el proceso de criticar, cuestionar y reflexionar de manera permanente.

La construcción de nuestros aprendizajes se desarrolló a partir de ocho encuentros. Mismos que iniciaron desde el mes de mayo de 2012 hasta el mes de noviembre de 2012. Supuso, comenzar identificando con los niños(as) el tipo de actividades que les interesaba con el fin de efectuar prácticas educativas coherentes a ellos(as) (Peralta, 2002). Con esta idea se organizaron los tres primeros encuentros. 
El primer encuentro inicia con una conversación reflexiva entre docentes, con la intención de solicitar un espacio para proponer talleres donde fueron las y los niños quienes decidían qué querían realizar. Por la cultura institucional, en este Jardín de Niños la planificación es un elemento establecido que se diseña de manera colegiada entre todas las docentes y no puede modificarse, sin embargo, sí es posible crear estrategias diversas articuladas con lo planificado. A raíz de esta conversación inicial con la docente a cargo del grupo, me preguntaba:

- ¿Y a los niños(as) se les está respetando?

- ¿Qué pasa si no se cumple con la planificación?

- ¿Cómo afectan mi formación profesional y mis creencias la dinámica de aula?

- ¿Cuál es mi rol ante la planificación y los niños(as)?

- ¿Qué pasa, si construimos una dinámica de aula donde todos estos elementos se articulen de manera activa, flexible y coherente con el desarrollo de los niños (as) incluyendo su visión?

- ¿Podrá provocarse algún cambio en nuestras actuaciones?

Desde mi papel de docente-investigadora esta primera conversación me confrontó con mi propia práctica como maestra de preescolar, con mis ataduras, miedos y desaciertos, constituyéndose entonces, en el primer eslabón en la escritura de un nuevo rol para mi vida pedagógica, cabe mencionar que vivir este proceso generó en mí la posibilidad de aprender a asumir dos roles el de investigadora y retomar el que hace muchos años venía desempeñando el de docente de preescolar, claro está desde posturas y visiones completamente nuevas. Además de lo anterior también inicie por cuestionarme respecto a mis años de experiencia y mi formación como maestra, me encontré frente al espejo sin reconocer el por qué de mi accionar en el pasado. Esto es precisamente lo que me permite la investigación- acción, el desarrollo de habilidades para reflexionar en torno a mi práctica (Elliot, 2005). 
Seguidamente, después de esta primera conversación entre la docente a cargo del grupo y yo, se tomó la decisión de permitir a los niños (as) elegir la actividad que quisieran hacer, sin embargo esta elección giró en torno a un tema específico dado por la docente (las frutas), así inició esta primera experiencia con los y las estudiantes, cabe mencionar que cada encuentro fue propuesto como un espacio más para el juego, la actividad que eligieron consistió en la elaboración de un helado de frutas, la participación de los niños (as) comenzó por pensar en ¿qué hacer?, la toma de decisiones, los acuerdos (votar para elegir la mejor actividad) y desacuerdos entre pares (la actividad más votada fue la que se realizó, se respetó la voluntad de la mayoría aunque no fuera la que algunos deseaban), la responsabilidad de cada uno por realizar la acción que decidió hacer (pelar frutas, colaborar en la limpieza, repartir ingredientes o materiales, etc.). Concebido desde la visión del niño (a) como actor principal del proceso educativo y la premisa de aprender haciendo, descubriendo y experimentando), encuentro que tuvo diferentes intencionalidades una de estas fue ir descubriendo sus intereses y necesidades, además ir identificando nuevos roles docentesestudiantes asumidos por asumir.

Finalizada la actividad con los y las estudiantes se abrió nuevamente un espacio para una segunda conversación entre ambas, por medio del dialogo, la acción, la observación y la reflexión se intentaba generar una comprensión y percepción diferente de los roles que cada uno había asumido. Es evidente que este primer encuentro se convirtió en una experiencia de aprendizaje para todos (as), abrió un espacio en la rutina diaria, dio lugar a un mayor disfrute basado en la toma decisiones, el respecto en las interacciones personales, la escucha, el compartir, el devolver la palabra y la mirada al otro, descubrir que la realidad es dinámica y cambiante. Conscientes de que educar nos exige un cambio, responsabilidad y compromiso en nuestra capacidad de acción (Freire, 2006). Los hallazgos más importantes en este primer encuentro refieren a que necesitamos: 
- Brindar la oportunidad de descubrir y conocer el tipo de actividades que interesan y motivan a niños y niñas.

- Abrir espacios para la expresión de opiniones, deseos, experiencias, preguntas. Se trata de experimentar cosas nuevas, construir conocimientos, participar y disfrutar.

- Enfocar el rol docente hacia cuestionar su quehacer (observar, dialogar, analizar, participar con entusiasmo, compartir, a integrarse, disfrutar y aprender).

- Intensificar la participación de la docente a cargo del grupo en el proceso, reconstruir en conjunto un lugar de participante y no de espectadora de su realidad.

Nuestra búsqueda continuó y nos aventuró a un segundo encuentro, producto de la reflexión derivada de la segunda conversación, sustentada en la primera experiencia de acción entre niños(as) y docentes surgieron comentarios como:

- "Les da la oportunidad de expresar sus deseos, criterios e ideas y a nosotros de escucharlos. Se hace participativa la clase, ya que no solamente las actividades son dirigidas por la docente. La clase se desarrolló en forma muy interactiva, porque ellos y ellas fueron los que realizaron la actividad (...) La manera que ellos y ellas están comentando con sus padres las actividades y el apropiarse de la frase nosotros y nosotras decidimos e hicimos" (docente a cargo del grupo).

- "Si nosotros estamos proponiendo el tema ellas y ellos no están eligiendo libremente, lo que tenemos que hacer es guiarlos para que se respeten los criterios de la mayoría, porque van a surgir muchos temas. Entonces hay que dejarlos que ellos y ellas elijan el tema y tratar que se respete el criterio de la mayoría y motivarlos que aunque no era lo que algunos querían se tiene que respetar" (docente a cargo del grupo).

Sin duda alguna, son esas frases las que marcaron una nueva ruta en el camino, para este segundo encuentro, no se les dio un tema específico como en el anterior, la intencionalidad fue que ellos (as) 
eligieran libremente de acuerdo a sus posibilidades la actividad que quisieron hacer y conocer basados en sus intereses, permitiéndoseles una nueva posibilidad de aprendizaje, de búsqueda de nuevos roles, de toma de decisiones conjuntas, de ir descubriendo la relación entre la palabra, la acción y la reflexión (Freire, 2006).

Con la experiencia anterior presente y el no darles un tema específico generó un nuevo espacio para intensificar las participaciones, la toma de decisiones y el cuestionamiento, de esa manera dio inicio este otro encuentro con la pregunta ¿en esta ocasión que quieren hacer? Sin dales ningún tema específico surgieron muchas ideas en la medida en que eran mencionadas se les cuestionaba ¿se puede cocinar dentro del aula? ¿Qué necesitamos para realizar esa actividad? ¿Pensemos en actividades que si se puedan realizar acá en el Jardín?, después de tomar muchas decisiones (el voto por las actividades más aceptadas por la mayoría, los materiales que se necesitaron, algunos acuerdos) finalmente la actividad por realizar fue una piñata, en este encuentro jugamos a hacer piñatas las que fueron utilizadas para celebrar el cumpleaños de algún hermano (a), invitar a primos y amigos a reventarlas, pedir a papá o mamá comprar dulces y sobre todo demostrar que ellos (as) si podían hacer piñatas.

Como investigadora y docente esto me llevó a pensar que el rol que desempeñamos puede cambiar en cualquier momento, pasar de uno impositivo en el cual el docente es el responsable de proponer las actividades y las ideas, a uno más flexible que genere espacios donde las construcciones de las mismas sean en conjunto (niños (as)docentes), permitiendo la autonomía, la toma de decisiones, el pensamientos, el disfrute y la acción, sin embargo todo lo anterior depende de la situación en la que nos encontremos y las acciones que estemos dispuestos a desarrollar y cambiar. El cambio paradigmático puede iniciar con la reflexión pero debe concretarse en la acción. Acción y reflexión constituyen un solo proceso en continuidad. 
Momento a momento la participación de los niños (as) en las actividades que estaban proponiendo realizar en el aula, se fueron intensificando, se infiere el gusto por asumir roles activos, desde los cuales enfrentan conflictos que enfrentan entre autonomía y dependencia. Su progresiva comprensión de cómo sus decisiones son tomadas en cuenta se traduce en su capacidad de esperar su turno, manejar acuerdos, respetar a los demás, tener paciencia, opinar, participar en las decisiones del grupo. De igual manera, para nosotras como docentes es una oportunidad para cuestionar el rol asumido ante la generación de actividades planificadas.

Una vez que finalizamos esta nueva experiencia de aprendizaje (hacer piñatas), nuevamente se abrió un espacio de análisis y reflexión entre docentes, tratando de incorporar e integrar lo que íbamos descubriendo. Las percepciones de ambas destacan la capacidad de los niños (as) de tomar decisiones en un ambiente de respeto mutuo, de participación con entusiasmo en las actividades, de interesarse por aprender haciendo. Elementos que coinciden con la necesidad de generar procesos donde se pueda reflexionar conscientemente el rol que tenemos las personas adultas. Mata (2007) menciona que el compromiso con el cambio, se promueve al facilitar espacios, siempre discriminando qué es lo que hay que cambiar y qué debe hacer cada sujeto. Poco a poco nos acercamos paulatinamente a ello.

Desde mi papel de docente investigadora, el cuestionamiento pasa a ser un hábito que disfruto. Me pregunto ¿Qué se puede leer en la participación de los niños (as)? ¿A qué me están invitando? Se me presenta como reto la capacidad de ver más allá de la palabra y la acción. Me hago consiente de la responsabilidad que tenemos como docentes de remirar y replantear lo que estamos haciendo, lo que los y las niñas hacen y desean hacer, en un espacio de respeto mutuo. En este sentido, mi papel como investigadora además de observadora participante y cuestionadora, me llevó a contribuir junto con la docente a cargo del grupo a construir un rol más circular, democrático en relación con las y los estudiantes, a disfrutar con 
ellos y a descubrir estrategias para que nuestras intervenciones respondan a intereses y necesidades que manifiestan.

En la medida que avanzaba este proceso, producto del dialogo, la acción y la reflexión nos damos cuenta que como docentes necesitamos remirar nuestras prácticas educativas, cuestionar nuestros actos, percepciones y roles asumidos. Abiertas a nuevas reflexiones y acciones, nos entregamos a un tercer encuentro, siempre desafiante siempre fascinante. Abrimos nuestra mirada a una nueva experiencia, proponiendo un cambio de estrategia metodológica, la cual consistió en la organización de un taller de juegos por las docentes en aras de satisfacer los intereses y las necesidades que se habían ido identificado en el proceso, destacando el papel fundamental que tiene el juego en el desarrollo integral, además de permitir espacios de disfrute, de diversión, de movimiento, de compartir, de participar, de aprender y de relacionarse. En la búsqueda de generar mayores oportunidades de construcción de conocimientos, se vuelve indispensable el cuestionamiento, la duda y la incertidumbre, bajo estos supuestos y una pregunta se organizó este encuentro ¿podemos responder a los intereses y necesidades de los niños (as) desde una propuesta pensada en y para ellos (as), sin tener que ser los y las estudiantes las que elijan la actividad que quieren realizar?

Una vez más los niños (as) asumieron un papel protagónico, cabe destacar que en esta ocasión no fueron ellos (as) los que eligieron las actividades que se estaban realizando, pero sí, estas fueron propuestas con una intencionalidad clara de un adulto, el juego como un elemento indispensable en el desarrollo integral basadas en los conocimientos reconstruidos, donde todos (as) estábamos aprendiendo a aprender de lo que ya sabíamos, a construir nuevos conocimientos estableciendo relaciones entre la teoría y la práctica, asumiendo posturas diferentes desde el pensar y hacer lo que nos lleva a efectuar prácticas pedagógicas más consientes. Retomando la vivencia pudimos reflexionar acerca de la necesidad que tienen las y los estudiantes de experimentar movimiento, libertad y 
oportunidades de creación. Para lo cual esperan que los docentes quitemos estructuras y escuchemos lo que piden sin hablar, disfrutemos y sintamos con ellos y ellas, al tiempo en que promovemos la libertad y el aprendizaje.

Articulando los hallazgos evidenciados hasta este momento del proceso investigativo, queda claro que la intervención docente que responde a intereses y necesidades de los niños (as) es aquella que:

- Los tiene claramente identificados.

- Mantiene el papel protagónico de los niños (as) como constructores que aportan a las actividades que se van a realizar.

- Promueve una planificación docente intencionadamente organizada para responder a intereses y necesidades.

- Se organiza la intervención docente desde el conocimiento del niño(a), del desarrollo humano y de la actitud propositiva.

Además supone la necesidad de docentes que sean capaces de:

- Acompañar, escuchar y dialogar.

- Disfrutar y aprender.

- Promover espacios de libertad.

- Observar y reflexionar.

- Investigar y cuestionar.

- Tener claridad en qué y cómo se hace.

Ahora bien, como docente investigadora, estos tres primeros encuentros desarrollados, tanto en su dimensión de planificación y análisis con la docente a cargo del grupo, como con la acción con niñas y niños, constituyeron la oportunidad para liberarme de mis "certezas" y "recetas", reflejarme en un espejo, pensar y sentir que no quiero seguir actuando de la misma manera. En este sentido, puedo decir que mi rol cambia hacia una docente que dialoga, comparte, analiza y percibe una dinámica de aula desde diferentes 
visiones.

Sumado a lo anterior, viendo el cambio como una oportunidad para crecer y aportar, dispusimos vivir un cuarto encuentro. Cabe mencionar que el mismo respondió a un giro en mi percepción del proceso, como se menciono anteriormente la intención metodológica inicial fue entrar a la dinámica de aula por medio de talleres, porque pensaba que esa estrategia era la más pertinente al movimiento natural de los niños (as) y que bastaría eso para producir cambios. Sin embargo, se evidencia que debemos trascender, se vuelve indispensable profundizar en nuestra comprensión del rol docente y la percepción del acto educativo, es decir organizar o planificar las intervenciones es tan importante como ejecutarlas.

Se puede decir, entonces que, en eso consistió esencialmente el giro en el proceso de investigación, no concentrarse más en el hacer con los niños (as) sino en el cómo, en la manera que la docente a cargo del grupo organiza su práctica para responder a los intereses y necesidades de las niñas y los niños, considerándolo como el aspecto vital por comprender. En este sentido, se vuelve necesario entender la práctica docente como una praxis social, objetiva e intencional en la que intervienen los significados, las percepciones y las actuaciones de los agentes en el proceso y de esta manera, ir enriqueciendo tanto el "saber" como el "hacer" pedagógico (Peralta, 2002). Las percepciones y haceres docentes determinan el tipo de dinámica de aula que se desarrolla.

Así, a partir del cuarto encuentro, la intencionalidad se dirigió en profundizar el análisis considerando dos aspectos principales:

- La identificación de lo que se hace diariamente en el aula de clases mediante registro audiovisuales, con la intención de remirar el rol docente.

- La comparación de lo observado en la rutina diaria versus lo acontecido durante los tres talleres lúdicos iniciales. 
A medida que profundizábamos en el análisis de lo que acontecía diariamente en el aula versus la lista de los intereses, necesidades y potencialidades identificadas en el grupo, reflexionamos que:

- Conocer sus intereses y necesidades sirve para proponer actividades que a ellos les gusten y les motiven.

- Es necesaria una docente que: acompañe, promueva, pregunte, disfrute, respete y participe con y junto ellos (as).

- Se trata de que nos escuchemos, hablemos, observemos y nos respetemos.

- Los docentes necesitamos un espacio o una posibilidad para observarnos en lo cotidiano, que nos permita cuestionar nuestras actuaciones desde una posición crítica y creadora de nuevas oportunidades de aprendizaje (docente investigadora).

Al abrirnos a nuevas formas de ver lo que acontece diariamente en el aula, cuestionábamos cómo se están sintiendo los y las niñas, si en el aula se les están respetan sus intereses, se satisfacen sus necesidades y potencialidades, qué estábamos sintiendo y viendo las docentes, reflexión que llevó a la docente a cargo del grupo a exteriorizar:

"muchas veces es necesario que alguien externo acompañe los procesos dentro del aula para que pueda ver cosas que se pueden mejorar, que muchas veces no se está consciente que se pueden cometer errores pero que se puede cambiar".

Retomar un proceso vivido requiere de una reflexión constante acerca de la práctica que se realiza, con el propósito de tomar las mejores decisiones, retomar lo que ha sucedido, ver lo invisible, conocer lo desconocido y objetivarlo en la realidad (Jara, 1994). Así como, la convicción que educar significa generar entusiasmo, que todos (as) se sientan vivos, se diviertan en una aventura lúdica compartida, sentir y hacer sentir, participar entregando lo mejor de sí y recibiendo lo mejor de los otros son las premisas que me inspiran 
(Gutiérrez, 2004).

La reflexión entre las docentes continuaba, dando paso al quinto encuentro, cuya intencionalidad nuevamente fue el análisis crítico de la propia práctica, mismo que posibilitó reflexionar acerca de nuestras intervenciones, decisiones, roles y la participación de los niños y niñas en las actividades propuestas. A partir de estas reflexiones, como docente investigadora decido que puedo aportar al proceso si me focalizo en la construcción de criterios a considerar para el diseño de actividades en del aula.

Propongo en ese momento a la docente a cargo del grupo, avocarnos a la construcción de los criterios para el diseño de las actividades que se van a planifican para los y las niña. Propuesta que nos llevó a promover la responsabilidad, autonomía, sentido crítico, capacidad de tomar decisiones, búsqueda de soluciones a los problemas, pero sobre todo favorecer la creatividad. Como argumenta Mata (2007) la persona que educa conforme transcurre el tiempo puede asumir cierto escepticismo sobre las posibilidades reales de aplicar lo aprendido en su formación inicial, por lo cual, debe hacer un esfuerzo hacia la madurez profesional que le permita discriminar entre lo posible, lo conveniente y lo que está en capacidad real de hacer. Eso es lo que hicimos en este quinto encuentro.

Con esas ideas presentes, iniciamos nuestro análisis destacando primeramente a que intereses y necesidades se estaba respondiendo con cada una de las actividades que se habían planificado para los y las niñas. Permitiendo de esa manera, reconocer algunas limitaciones y permitiéndonos crear oportunidades para la mejora. Nos hicimos conscientes de esto. Como señala Freire (2006) la conciencia es el primer paso para cualquier transformación. Cada momento de este encuentro, nos permitió entender, conocer, revivir, pero sobre todo arriesgarnos a cuestionar lo que hemos hecho. Con lo cual, progresivamente descubrimos respuestas a las preguntas ¿qué debo hacer mañana? ¿Cómo puedo cambiar? El examinar la propia práctica desde una mirada estricta, con el fin de 
mejorar, se construye sobre un repertorio propio que conjuga la reflexión en y sobre la acción (Olson, s.a.).

En este sentido mi intención como docente investigadora fue propiciar espacios para que la docente a cargo del grupo reflexionara en torno a su actuar, pero en el fondo, fueron cada uno de esos espacios, los que me permitieron cuestionar mi actuar, precisamente porque la investigación-acción permite involucrarme, compartir, escuchar, mirarme en los otros y aprender. Como docentes reflexionábamos que generar una dinámica de aula flexible, donde cada uno puede construir procesos de aprendizaje desde el ser, el saber y el hacer, partiendo de modificaciones en los roles asumidos entre docentes-estudiantes, docente-planificación, con un fuerte componente de reflexión, posibilita cambios en el hacer, pero con más intensidad transformaciones en nuestra construcción paradigmática de la realidad.

Es así que, el pensar críticamente puede contribuir a replantearnos y a liberarnos de prácticas irreflexivas (Freire, 2006) bajo esta pensamiento nos dispusimos a seguir avanzando en nuestro análisis y transformación, nuestro sexto encuentro, representó una continuidad del anterior con la intencionalidad de profundizar en el análisis del papel de la docente a cargo del grupo ante los intereses, las necesidades y las potencialidades de niños (as), mismo que fue iniciado, como en los anteriores encuentros con una conversación, de donde se desprendían cuestionamientos, decisiones, observaciones y vivencias.

Nuestra reflexión continuaba, más preguntas que respuestas, era esa actitud cuestionadora la que permitía profundizar en nuestra conciencia en torno al acto educativo del que estábamos siendo parte. Como señala Peralta (2002) el rol del educador es clave y debe partir del propio análisis se su actuar, si quiere convertirse en un verdadero agente de transformación. Producto de la sistematización y análisis de la información construida entre docentes durante los encuentros 4, 5 y 6 , concluimos que podemos 
re-definir nuestro actuar encaminándolo hacia:

- Las transformaciones de las acciones cotidianas.

- La planificación basada en intereses, necesidades y potenciales identificados.

- La reflexión recursiva que nos permite ir escribiendo el nuevo rol que asumiremos.

- La profundización de respuestas en torno a las preguntas ¿cómo hacer una práctica pedagógica respetuosa de los niños (as) sus intereses y necesidades? y ¿cómo se hace la planificación y bajo qué criterios se elabora?

Es momento de tomar la decisión de generar una organización de la dinámica de aula diferente, partiendo de los aprendizajes construidos, es lo que abrió el espacio para el séptimo encuentro y nos aventurábamos a reflexionar acerca de nuestra planificación, bajo la convicción que pensar acertadamente exige una reflexión crítica sobre la práctica (Freire, 2006). Con la disposición de hacer una lectura consciente de los criterios que a lo largo del transitar por esta nueva forma de aprendizaje fuimos construyendo, propongo entonces a la docente a cargo del grupo retomar elementos de todos los encuentros anteriores y enunciamos:

- Criterios a tomar en cuenta antes de proponer actividades.

- Las condiciones que deben presentar dichas actividades si queremos que respondan a intereses, necesidades y potencialidades del grupo.

Sobre la base de nuestras construcciones destacábamos que, en relación criterios a tomar en cuenta antes de proponer actividades estas deben: potenciar al máximo el desarrollo integral, la participación y el rol activo, favorecer mediante la interacción la construcción de aprendizajes. Mientras que en relación con las condiciones es importante mencionar que las actividades diseñadas deben: enfocarse a potenciar el desarrollo integral, propiciar y fomentar la participación activa en la palabra y la acción a los niños 
(as) y ocurrir en espacios que permitan la libertad de movimiento.

Como docentes somos conscientes que las características, necesidades e intereses y potencialidades de las y los estudiantes constituyen el punto de partida de toda planificación y que la misma debe visualizarse como una aplicación flexible, ofreciendo diferentes posibilidades y alternativas tanto al planificarse como al aplicarlas. Decidimos aprovechar las construcciones anteriores de los criterios a tomar en cuenta antes de proponer las actividades para los niños (as) y condiciones que deben presentar las mismas y abocarnos a generar una propuesta de planificación intencionada, en la cual cada actividad tenga sentido para quienes la van a realizar, implicando situaciones placenteras que posibiliten actuaciones efectivas. Enfatizamos que los propósitos de la planificación de las actividades diseñadas e implementadas deben encaminarse a:

- Favorecer el desarrollo integral del niño (a).

- Promover la construcción de conocimientos por medio de la interacción entre docente-niño.

- Promover el juego y la participación activa para todos (as).

- Promover actividades que ofrezcan a los niños (as) oportunidades para desarrollar su confianza en sí mismos, autonomía, creatividad, socialización, conocimientos.

Así como también, describíamos algunas ideas acerca del rol que debe asumir la docente durante las actividades diseñadas e implementadas con los niños (as). Estas ideas son el resultado tanto de la conversación como de los aprendizajes que hemos construido respecto a este componente en encuentros anteriores, propusimos entonces que el rol docente que íbamos a asumir en el momento de ejecutar las actividades que se estaban planificando llevar a cabo en la siguiente semana de clases en el Jardín de Niños, van enfatizar en:

- Observar.

- Presencia actuante y motivante.

- Incentivar la participación grupal e individual. 
- Disfrutar junto con los niños (as) de las actividades.

- Aprovechar las diferentes actividades para cuestionar.

- Propiciar la interacción grupal.

Finalmente cabe mencionar, que planificar juntas y luego valorar los resultados de dicha planificación, también de manera conjunta, fue una gran oportunidad de aprendizaje y reflexión, por todo lo que ese proceso generó en mí. Se puede afirmar que en este encuentro, fuimos testigos de cómo la mediación docente inició desde el momento que hipotetizabamos en cómo sería nuestra clase, qué haríamos en ella y para qué. Es decir, desde que estuvimos pensamos y construyendo nuestra propuesta de planificación. No cabe duda, que fuimos tomando decisiones, reflexionando, construyendo, descubriéndonos como seres capaces de cambiar y trascender, que siempre que tengamos un ¿Por qué? encontraremos un ¿Qué? y un ¿Cómo?

En nuestra conversación, es particularmente clara la idea de que cada acto puede influir en las diferentes dimensiones del desarrollo del niño, comprendiendo esto como abordaje integral. Quizá la disyuntiva que enfrentamos como docentes refiere a cómo llevar estas premisas conceptuales a la práctica, y hacerlo de manera sistemática y permanente. En este sentido, los criterios a tomar en cuenta antes de proponer las actividades y las condiciones que deben presentar las actividades diseñadas e implementadas con el grupo de niños y niñas, identificados en el Encuentro 7, se visualizan como una de las herramientas que hace posible esta articulación.

Freire (2006) menciona que educación es reflexión y acción sobre el mundo para transformarlo, de manera que, no puede haber palabras verdaderas si no van juntas con la acción, el decir y el hacer que es transformar la realidad. Como docentes nuestro primer gran reto es articular decir y hacer, en tanto podemos por un lado mostrar una actitud abierta y disponibilidad para cambiar la organización de nuestro hacer y por el otro, dejarnos vencer por el peso de una rutina y la comodidad, asumiendo el lugar más peligroso para un educador: 
el "etiquetado paradigmático".

Desfamiliarizar lo cotidiano, evitar la ceguera de la costumbre es el segundo gran reto que toda docente debe enfrentar, de lo contrario podemos permanecer imperturbables cognitivamente, debemos mantener nuestra práctica cuestionada, abierta y sujeta a la incertidumbre. Las buenas ideas, inclusive, siempre deben estar siendo sometidas a la práctica reflexiva y participativa para probar su pertinencia.

De esa nueva experiencia de organización, diseño e implementación de las actividades emerge un octavo encuentro cuya intensión fue retomar los resultados de la planificación conjunta. Sin embargo, no fue posible para la docente a cargo del grupo ejecutarla en la práctica, así que abrimos un espacio de conversación final, fundamentada en la pregunta ¿cómo le fue con el desarrollo de la planificación? ¿Se presento alguna dificultad al desarrollarla? ¿Por qué no la pudo hacer? más que respuestas estaba buscando encontrar explicaciones, esperaba cambios y transformaciones en los demás y no me estaba permitiendo interiorizar que eso es producto de decisiones personales, que nadie es el responsable por generar cambios, además descubro que ambas transitamos por el camino de pregunta, ambas finalizamos este recorrido, muchos aprendizajes quedan, al igual que incontables preguntas abiertas que estoy segura que seguiré contestando con la particularidad de cada grupo educativo del que forme parte, Algunas de esas cuestionantes que surgieron de este proceso son:

- ¿Quién es el responsable de los procesos educativos?

- ¿Qué es ser un docente?

- ¿Cómo continuar soñando y construir realidades educativas diferentes?

- ¿Cuál es el rol de un docente?

- ¿Cómo co-aprender con los estudiantes?

- ¿Cómo crear un equipo de trabajo docente abierto a la transformación? 
- ¿Qué criterio y condiciones son los mejores de considerar en el proceso de planificación con cada uno de mis grupos de trabajo?

- ¿Cómo asumir la planificación como un mapa, una hipótesis y no como una realidad construida?

- ¿Cuáles son los elementos indispensables para crear una dinámica de aula respetuosa de los niños (as), sus intereses y necesidades?

- ¿Qué es lo que hace difícil modificar los roles docentes asumidos?

Como docente investigadora no pude dejar de tener sentimientos encontrados, ilusiones y desilusiones, pero siento que es la riqueza que permitió vivir este proceso de investigación-acción. Estoy consciente de que nuestra transformación depende de la posición que asumamos, del momento vivido, de la realidad que queramos construir, de las experiencias propias, de la comprensión que tengamos acerca del quehacer cotidiano, el cómo nos veamos a nosotros mismos es la herramienta con la construimos el mundo. Además aprendí a darle tiempo al tiempo, a esperar el momento, porque todo llega cuando tiene que llegar, el primer paso está dado, cuando menos lo esperaba la vida me colocó frente a un desafío, poniendo a prueba mi coraje y voluntad de cambio, con estas ideas presentes continúo en transformación... 


\title{
Reflexiones finales: los cambios que provoca la investigación- acción en el ser y hacer de una docente
}

\author{
El profesor no debe seguir siendo objeto de investigación \\ de personas externas, sino un investigador de símismo, \\ pero para poder llegar a ello, \\ debe empezar por pensar y preguntarse
}

Stenhouse (1998).

Desde un postura reflexiva, como ya he mencionado, esta investigación parte del ser y del actuar como docente, siempre que hablo, pienso o analizo el rol de los otros(as), estoy hablando de mí como docente de preescolar e investigadora, cuestionándome al reflejarme en la mirada del otro. Ejercicio que me resultó muy interesante, tanto que aprendí interactuando y compartiendo. En realidad me sorprende como el tiempo para observar y conversar permite profundizar nuestros saberes. Sin lugar a duda, no podía ver, escuchar y dialogar con el otro, si no me miraba, escuchaba y dialogaba conmigo misma. Esto es precisamente lo que permitió en mí la investigación- acción, el desarrollo de habilidades para reflexionar sobre la propia práctica a partir de la comprensión de los problemas que la rodean (Elliot, 2005).

Resulta importante mencionar que, la transformación del quehacer educativo inicia por el cuestionamiento y reflexión, los cuales, dirigen a cambios que pueden resultar difíciles, pero no imposibles y que exigen una actitud permanente de investigación e innovación (Freire, 2005). Además, invitan a pensar y actuar de manera diferente.

Para lo cual, surge la necesidad de ir en busca de un estilo de docente que responda asertivamente al paradigma naturalista, por ende a nuevos roles, concepciones sobre estudiantes-docente, a las demandas y los desafíos que la realidad impone y que obligan a una revisión permanente del quehacer educativo, que lo lleven a generar teorías y prácticas cada vez más pertinentes a las necesidades y el 
derecho que tienen los niños de recibir una educación oportuna (Peralta, 2002).

La intencionalidad de esta investigación fue generar espacios y acompañar a una docente en un viaje hacia la transformación de sus pensamientos, visiones, percepciones y acciones, sin estar consciente que la transformación principal era mía como docente e investigadora. Toro (2005) indica que

para que lo vital surja algo ha de abrirse (...) de manera similar los corazones han de abrirse para hacer posible el florecimiento de las acciones de la vida. El gesto de abrir no basta, implica firmeza y valentía porque abrir siempre supone un exponerse, un entregarse, un arriesgarse. Abrirse conlleva un riesgo porque cuando me abro me muestro en lo que soy, entonces puedo volverme vulnerable ( $p$. 67).

Sin lugar a dudas, como investigadora me aventuré a dejar que las cosas surgieran, a vivirlas, gozarlas, disfrutarlas y permitirle a los otros(as) hacerlo junto a mí. Conforme avanzaba, surgían nuevos acontecimientos, que me exigían no solo mirar la realidad, sino también compromiso, pasión, cambios, que me permitían incorporar representaciones y conceptos nuevos que reconstruían o sustituían los anteriores.

Las ideas son grandes cuando son aplicadas, es decir, cuando se transforman en acciones. De manera que, asumir un rol activo supone sentir, pensar y actuar, aceptar que somos responsables de nuestros aprendizajes, que no solo se aprende de la mano de los demás, sino que los demás aprenden de tu mano. En especial cuando el diálogo, la observación, la reflexión y el cuestionamiento son las herramientas utilizadas para desarrollar conciencia acerca de nuestros propios actos en el aula.

Sumado a lo anterior, en la medida en que avanzaba esta experiencia, me re-definía y cuestionaba ¿Quién soy como docente? ¿Cuáles son mis miedos o limitaciones al organizar mi quehacer pedagógico? ¿Soy 
consciente de lo que está ocurriendo a mí alrededor? ¿Qué está generando en mí este proceso? ¿Debo cambiar mis intervenciones pedagógicas? ¿Por qué debo hacerlo? ¿Siento la necesidad de vivir un proceso de transformación? El compromiso con el cambio, se promueve al facilitar espacios para la reflexión, mismos que posibilitan sacar a la luz aciertos y desaciertos, responsabilidades, decisiones, pero sabemos que todo ello implica un esfuerzo por vencer la resistencia al cambio (Mata, 2007).

Desde ese nuevo posicionamiento, pude compartir mi crecimiento personal, viéndose reflejado principalmente en las interacciones como docente e investigadora y que fueron la sumatoria de un constante mirar-remirar, pensar-repensar y actuar. Considerando que viví un proceso de construcción de aprendizajes sustentado desde las premisas donde: Cuestionando me cuestionaba, acerca de los roles desempeñados ante los y las estudiantes, la organización pedagógica, las percepciones o concepciones sobre educación, el perfil del educando que se quiere formar, la dinámica de aula, el rol de niños, niñas, padres de familia, la comunidad y mi responsabilidad como promotora del acto educativo.

En muchos momentos, hice un alto y reviví en mi mente situaciones específicas que había vivido a lo largo de mis años de experiencia como docente de preescolar, me atreví a juzgarme críticamente con la única intensión de mejorar y cambiar. Me hice consciente que solo por medio de esta vivencia podía transformar mi ser y hacer. Hoy acepto, vivo y digo que ningún acto verdaderamente educativo debe carecer de reflexión, esta investigación fue producto de ello; un intento de gritar en voz baja que tengo que repensar, observar al otro y aprender con el otro en una cadena sin fin.

No cabe duda que estas palabras marcan una gran diferencia entre lo que se hace y lo que se dice, para lo cual el arte de la pregunta es la llave del constante saber para un investigador, invitándole a pensar, dialogar, buscar respuestas a nuevas situaciones Podemos iniciar con preguntas como ¿Qué quiero? ¿Para qué y por qué lo 
hago? ¿A dónde quiero llegar? ¿Estoy respondiendo a...? ¿Hago lo oportuno? ¿Qué debo cambiar? ¿Cómo se puede lograr un cambio...? ¿Cuál es mi rol frente al aprendizaje?

Observando me observaba, me asumía como una docente que no puede prescindir del acto de mirar-escuchar para iniciar un proceso autoreflexivo, que necesita "ir y venir" las veces que sean para ver lo que mis ojos se han negado a ver y mis oídos se niegan a escuchar. Debo estar atenta ante cualquier situación. Todo es relevante, es importante, cualquier momento es el mejor, una posibilidad de ser, crecer, aprender, vivir y convivir. Hay que tener tiempo y paciencia para sostener la mirada y escuchar qué nos dice eso que vemos (Toro, 2005).

Aprender es un juego siempre inacabado y en conexiones, bajo esta premisa también transité como docente e investigadora, me di cuenta que no se trata de alcanzar respuestas sino de formular preguntas. Como docente este proceso me permitió reconocerme con nuevas emociones que exigen nuevas miradas, acciones, visiones, decisiones, presencias y proyectos. Como investigadora me reconozco desde una actitud aprendiente permanentemente, vivo en la incertidumbre y lo disfruto, gozo con los cambios, me atrevo a soñar con un mañana mejor. Me cuestiono y valoro que soy una persona diferente en mí sentir, pensar y hacer. En este momento puedo decir que no soy la misma docente, que mi transitar por la observación, acción y reflexión transcienden, que soy libre, puedo abrir mis nuevas alas, volar y contagiar...

Finalmente, como nos dice (Olson, s.a) el convertirse en un docente investigador es transformar y transformarse en un sujeto que toma decisiones reflexivas y que actúa en consecuencia, que acepta la responsabilidad de esas decisiones, que crece, valora, es flexible, que se libera, que se atreve a vivir y dejar vivir, que aprovecha todo el recorrido como una permanente oportunidad de aprender, en esencia, no se puede trabajar sin reflexionar, ser docente supone inevitablemente un componente de reflexión fuerte, si deseamos 
construir el tipo de educación que planteamos, respetuosa de los niños(as) sus intereses y necesidades. En resumir, este fue camino transitado de las docentes en la búsqueda de sus propias transformaciones. 


\section{Referencias bibliográficas}

Dobles, Izaguirre y García. (2010). Investigación en Educación: Procesos, interacciones y construcciones. San José, Costa Rica: EUNED.

Elliot, J. (2005). La investigación-acción en educación. Madrid: Ediciones Morata.

Freire, P. (2005). La educación como práctica de la libertad. Buenos Aires, Argentina: Siglo XXI editores.

Freire, P. (2006). Pedagogía de la Autonomía: Saberes necesarios para la práctica educativa. Buenos Aires, Argentina: Siglo XXI editores.

Freire, P. (2007). Cartas a quien pretende enseñar. Buenos Aires, Argentina: Siglo XXI editores.

Gutiérrez, F. (2004). Pedagogía del aprendizaje: Germinando Humanidad. Guatemala: Save the Children.

Jara, O. (1994). Para sistematizar experiencias: San José, Costa Rica Centro de Estudios y Publicaciones ALFORJA.

Latorre, A. (2005). La Investigación Acción. España: Editorial Graó.

Mata, A. (2007). La Investigación- acción para la Formación continúa de educadores. San José, Costa Rica: UCR.

Olson, M. (s.a.). La investigación acción entra al aula. USA: AIQUE.

Peralta, M. (2002). Pedagogía de las Oportunidades. Santiago, Chile: Editorial Andrés Bello

Toro, J. (2005). Educar con “corazón”. Epaña:Editorial Desclée de Brouwer, S.A. 